\title{
BMJ Open Physician engagement in hospitals: a scoping review protocol
}

\author{
Tyrone Perreira, ${ }^{1,2}$ Laure Perrier, ${ }^{3}$ Melissa Prokopy, ${ }^{2}$ Anthony Jonker ${ }^{2}$
}

To cite: Perreira T, Perrier L, Prokopy M, et al. Physician engagement in hospitals: a scoping review protocol. BMJ Open 2018;8:e018837. doi:10.1136/ bmjopen-2017-018837

- Prepublication history and additional material for this paper are available online. To view these files, please visit the journal online (http://dx.doi. org/10.1136/bmjopen-2017018837).

Received 27 July 2017 Revised 7 December 2017 Accepted 18 December 2017

\section{CrossMark}

${ }^{1}$ Institute for Health Policy, Management and Evaluation, University of Toronto, Toronto, Canada

${ }^{2}$ Ontario Hospital Association, Toronto, Canada

${ }^{3}$ University of Toronto Libraries, University of Toronto, Toronto, Canada

Correspondence to

Dr Tyrone Perreira;

ty.perreira@utoronto.ca

\begin{abstract}
Introduction Literature on healthcare reforms highlights the importance of physician engagement, suggesting that it is a critical factor for lowering costs while improving efficiency, quality of care, patient safety, physician satisfaction and retention. As a result, many hospitals have adopted physician engagement as a top strategic priority, but little is known about the actual evidence, making it difficult for hospital leadership to identify relationships between true physician 'work engagement' and work outcomes. The aim of this scoping review is to identify factors associated with, and tools used to measure, physician engagement.
\end{abstract}

Methods and analysis This scoping review will be conducted as per Arksey and O'Malley (2005). The electronic databases that will be searched from inception onwards include MEDLINE, EMBASE and Cochrane Central Register of Controlled Trials. Grey literature will be searched via websites of relevant agencies such as Agency for Healthcare Research and Quality. Conferences and abstracts will be viewed and full paper requests made as required. Supplementary articles may be obtained by contacting field experts and searching references of relevant articles. All quantitative and qualitative study designs will be eligible that describe factors associated with, and tools used to measure, hospital physician engagement. After a small calibration exercise, screening and abstraction will be completed separately by two individuals, with discrepancies resolved by a third. Quantitative (frequencies) and qualitative analyses (generation of descriptives) will be conducted. Thematic analysis will be used to evaluate and categorise study findings.

Implications and dissemination This project is part of the Ontario Hospital Association's (OHA) initiative to improve its understanding of physician engagement. The review findings will be shared with all Ontario hospitals. Dissemination will occur through peer-reviewed publications and to the OHA membership through the $\mathrm{OHA}$ Learning and Engagement team.

\section{INTRODUCTION}

Physician engagement is of global interest. Literature on healthcare reforms highlights the importance of physician engagement, ${ }^{1}$ suggesting that it is a critical factor for improving physician satisfaction and retention, ${ }^{2}$ quality of care, patient safety, efficiency and lowering costs. ${ }^{2-4}$ As a result, many hospitals have adopted physician engagement as a top strategic priority, but little is known about the actual evidence to support these claims.
Strengths and limitations of this study

- This scoping review of physician engagement in hospitals is unique.

- This review is comprehensive, including all study designs with no time period or language restrictions.

- The paucity of literature addressing the process of engagement and the motivation behind engagement may limit findings, specifically factors associated with physician engagement or the tools used to measure physician engagement.

Traditionally, psychology focused on disease, damage, disorder and disability. Over the past century, this has been supplemented with an increasing interest in the term 'positive psychology', the scientific study of human strength and optimal functioning. ${ }^{5}$ This shift triggered attention in organisational psychology, with calls for "the study of positively oriented human resource strengths and psychological capacities that can be measured, developed, and effectively managed for performance improvement in today's workplace" (p. 698). ${ }^{6}$ One of the positive states that arose from this was the term 'engagement'. The term 'engagement' is defined as a positive, fulfilling work-related state of mind, which is characterised by vigour, dedication and absorption. ${ }^{7}$ Vigour denotes high energy, mental resilience, persistence and a willingness to invest effort in one's work. Dedication reflects a sense of pride, significance, enthusiasm, inspiration and challenge. Finally, absorption refers to deep engrossment in one's work, full concentration and difficulty detaching oneself from work whereby time passes quickly. ${ }^{7}$ Contrary to those who suffer from burnout, engaged employees have a sense of energetic and effective connection with their work activities, and they see themselves as able to deal well with the demands of their jobs. ${ }^{8}$

Over time, however, physician engagement has become a widely used term ${ }^{9}$ that encompasses different work attitudes and behaviours such as appropriate and effective 
use of hospital services, ${ }^{9}$ implementation of best practices, accountability, physician performance measurement, physician leadership development, enhanced communication, values alignment, ${ }^{4}$ policy advocacy, participation in research ${ }^{10}$ or involvement in strategy, decision making and care direction. ${ }^{9}$ This makes it difficult for physicians and hospital leadership to identify relationships between true physician 'work engagement' and work outcomes such as patient satisfaction.

There is a paucity of literature addressing the 'process' of engagement ${ }^{1}$ and the motivation behind physician engagement. ${ }^{11}$ Enhancing physician engagement requires understanding the underlying characteristics and values of engaged physicians ${ }^{1}$ and making an effort to better understand their world. ${ }^{12}$ In order to create work environments that motivate workers and support positive work outcomes, a clear understanding of the work psychology of hospital physicians is required. ${ }^{13}$

The aim of this scoping review is to identify factors associated with, and tools used to measure, physician engagement. The specific research questions are:

1. What factors are associated with physician engagement?

2. What tools identified in the literature measure physician engagement?

\section{METHODS}

\section{Commissioning agency}

This project was commissioned by the Ontario Hospital Association (OHA). Over the last 3years, the OHA has identified the hospital-physician relationship as one of its three areas of Thought Leadership. Over the past 2years, the OHA, in partnership with the Ontario Medical Association, has built a foundation to support the strengthening of hospital-physician alignment across the province. This scoping review will be conducted to inform OHA's work on strengthening hospital-physician alignment moving forward.

\section{Study design}

This scoping review will be conducted as per Arksey and O'Malley. ${ }^{14}$ This method involves five stages that include identification of the research question, identification of relevant studies, study selection, charting the data and finally collating, summarising and reporting the results. In addition, there is a parallel element whereby experts are consulted to inform and validate findings from the main scoping review. Scoping reviews are a knowledge synthesis approach used to map concepts in a particular area of research in addition to main sources and types of available evidence. ${ }^{15}$ Synthesising knowledge emphasises how the results from a single research study mesh with the larger body of knowledge on the topic. ${ }^{16}$

\section{Eligibility criteria}

Described below are the Population, Intervention, Comparator, Outcome and Study Designs eligibility criteria.

\section{Population}

Eligible studies will include physicians with hospital privileges working full time or part time. All specialties and subspecialties will be eligible, for example surgeons, hospitalists, oncologists and dermatologists. Studies examining trainees such as residents or fellows will be accepted into our review. If a study has a mixed group (eg, physicians, nurses, pharmacists, physical therapists, etc), only studies in which hospital physicians make up more than $50 \%$ of the population will be eligible in this review. All hospitals will be eligible (eg, public, private, urban, rural) as well as academic medical centres. All hospital units will also be eligible.

\section{Intervention}

Studies that examine engagement for hospital physicians will be eligible. For the purpose of this scoping review, we will be incorporating all aspects of engagement described above by Schaufeli et al, ${ }^{7}$ which include a positive, fulfilling work-related state of mind, characterised by vigour, dedication and absorption.

\section{Comparators}

All comparators will be eligible (eg, studies comparing different forms of engagement, or no engagement). Studies without a comparator will also be eligible (eg, studies that focus on barriers or facilitators of engagement).

\section{Outcomes}

Studies will be eligible that identify factors associated with hospital physician engagement and tools used to measure hospital physician engagement. Papers developing theory or frameworks will be excluded.

\section{Study designs}

All qualitative or quantitative methodologies will be eligible for inclusion, for example, experimental (randomised controlled trials, quasi-randomised controlled trials, non-randomised clinical trials), quasi-experimental (interrupted time series, controlled before-after studies), observational (cohort, case-control, cross-sectional, case series) and qualitative studies (interviews, open-ended questionnaires, focus groups).

\section{Time periods}

There will be no time period restrictions.

Setting

Eligible will be studies from any setting or country.

\section{Other}

All attempts will be made to translate papers not written in English, either by obtaining a translator or the use of Google Translate when feasible.

\section{Information sources and search strategy}

An experienced information specialist (LP) will search the following electronic databases from inception onwards: MEDLINE, EMBASE and Cochrane Central 
Register of Controlled Trials. The final literature search for MEDLINE can be found in online supplementary appendix A. Search results will be imported and stored in RefWorks (https://refworks.com) by an information specialist for reference management.

In addition to the aforementioned electronic search strategy, grey literature will also be searched via the websites of relevant agencies such as the National Institutes of Health, the Canadian Medical Association, GreyNet International, and Agency for Healthcare Research and Quality. Conferences and abstracts will also be viewed. If only conferences are available, the authors will be contacted to determine if full papers have been published. Due to the 3-month time frame, authors will each be contacted twice within a 2-week period. Supplementary articles may be obtained by searching references of relevant review articles and contacting experts in the field.

\section{Study selection process}

The results of the search will be uploaded to Covidence, a Cochrane technology platform to improve the production and use of systematic reviews for health (https://www.covidence.org/). This tool will be used for study selection (ie, title/abstract screening and full-text article screening) using the eligibility criteria outlined above. Differences in responses are flagged for reviewers, discussed and a final decision is agreed on. Any unresolved conflicts will be resolved by a third reviewer. Subsequently, all levels of screening and data abstraction will be conducted by two reviewers, independently.

\section{Data items and data abstraction process}

Data will be abstracted on study characteristics (eg, study design), population characteristics (eg, number of participants, country, physician specialty, hospital type, hospital unit/department), intervention characteristics (eg, engagement strategy, timing, required resources), tools used to measure physician engagement, intervention results (eg, barriers, facilitators, outcomes) and any factors reported to be associated with hospital physician engagement. Examples of 'factors associated with physician engagement' include, but are not limited to, demographics, characteristics in the work environment (eg, organisational support, quality of work-life and perceptions of safety), work attitudes (eg, physician work engagement, job satisfaction, commitment and empowerment) and work outcomes (eg, patient experience, safety, quality of care, individual and organisational performance).

A standardised Excel form will be used for data abstraction. A training exercise will be conducted first among the investigators using a random sample of 10 included studies. Conflicts will be discussed among reviewers until a final decision is agreed on. If poor agreement is found, revisions will be made to clarify the data abstraction form and the training exercise will be repeated. Subsequently, two reviewers will abstract data, independently, with conflicts resolved by discussion or a third reviewer if required.

\section{Synthesis of result}

Quantitative (frequencies) and qualitative analyses (generation of descriptives) will be conducted. Thematic analysis will be used to evaluate and categorise study findings, that is, factors associated with hospital-physician engagement and tools used to measure hospital-physician engagement. Meta-analysis will not be performed. Data will be charted. New categories will be added as required.

\section{DISCUSSION}

\section{Implications}

The OHA will use the results from this scoping review to strategically work with hospitals and our system partners to improve their understanding of physician engagement.

This project offers a cost-effective opportunity to save time and resources by identifying and sharing physician engagement research. Findings will help OHA members make evidence-based decisions, specifically where they may want to focus resources and research. This project will also help to advance the science in this area. To the best of our knowledge, no previous empirical studies have taken this approach.

\section{Dissemination}

This project is part of the OHA's initiative to improve physician engagement. This work is also informed by the OHA's Physician Provincial Leadership Council, which comprised senior physician leaders across the province to provide strategic advice to the OHA on various physician-related matters that impact hospital-based practice in Ontario.

The review findings will be shared with all Ontario hospitals. Results, however, may be of interest to hospitals globally who are seeking to improve their understanding of physician engagement and how it is measured internationally. Dissemination will occur through peer-reviewed publications and to the OHA membership through the OHA Learning and Engagement team.

Contributors TP and LP conceptualised the research and drafted the protocol. MP and AJ helped to develop the research idea, conceptualise the study and wrote sections of the protocol.

Competing interests None declared.

Provenance and peer review Commissioned; externally peer reviewed.

Open Access This is an Open Access article distributed in accordance with the Creative Commons Attribution Non Commercial (CC BY-NC 4.0) license, which permits others to distribute, remix, adapt, build upon this work non-commercially, and license their derivative works on different terms, provided the original work is properly cited and the use is non-commercial. See: http://creativecommons.org/ licenses/by-nc/4.0/

(c) Article author(s) (or their employer(s) unless otherwise stated in the text of the article) 2018. All rights reserved. No commercial use is permitted unless otherwise expressly granted.

\section{REFERENCES}

1. Denis JL. Exploring the dynamics of physician engagement and leadership for health system improvement: prospects for Canadian healthcare systems. Toronto: Institute of Health Policy, Management and Evaluation, University of Toronto, 2013. 
2. Jarousse LA. Physician engagement (getting your docs on board!). Hosp Health Netw 2014;88:41-7.

3. Milliken AD. Physician engagement: a necessary but reciprocal process. CMAJ 2014;186:244-5.

4. Scott CG, Thériault A, McGuire S, et al. Developing a physician engagement agreement at the Ottawa Hospital: a collaborative approach. Healthcare quarterly (Toronto, Ont. 2012;15:50-3.

5. Seligman ME, Csikszentmihalyi M. Positive psychology: an introduction. In: Flow and the foundations of positive psychology 2014. Netherlands: Springer. (pp. 279-298).

6. Luthans $F$. The need for and meaning of positive organizational behavior. J Organ Behav 2002;23:695-706.

7. Schaufeli WB, Salanova M, González-romá V, et al. The measurement of engagement and burnout: a two sample confirmatory factor analytic approach. J Happiness Stud 2002;3:71-92.

8. Schaufeli WB, Bakker AB, Salanova M. The measurement of work engagement with a short questionnaire: a cross-national study. Educ Psychol Meas 2006;66:701-16.

9. Spaulding A, Gamm L, Menser T. Physician engagement: strategic considerations among leaders at a major health system. Hosp Top 2014;92:66-73.
10. Frattaroli S, Webster DW, Wintemute GJ. Implementing a public health approach to gun violence prevention: the importance of physician engagement. Ann Intern Med 2013:158:697-8.

11. Kumar P, Sherwood A, Sutaria S. Engaging physicians to transform operational and clinical performance: McKinsey \& Company, 2013. Available at. http://healthcare. mckinsey. com/sites/default/files/ MCK_Hosp_MDSurvey. pdf (accessed 11 Apr 2016).

12. Rosenstein $\mathrm{AH}$. Strategies to enhance physician engagement. J Med Pract Manage 2014;31:113-6.

13. Kurtessis JN, Eisenberger R, Ford MT, et al. Perceived organizational support: a meta-analytic evaluation of organizational support theory. J Manage 2017;43:1854-84.

14. Arksey H, O'Malley L. Scoping studies: towards a methodological framework. Int J Soc Res Methodol 2005;8:19-32.

15. Tricco AC, Lillie E, Zarin W, et al. A scoping review on the conduct and reporting of scoping reviews. BMC Med Res Methodol 2016;16:15

16. CIHR. A guide to knowledge synthesis. 2010. Available at http:// www.cihr-irsc.gc.ca/e/41382.html (accessed 19 Sep 2017). 